\title{
INVARIANT SUBSPACES OF FINITE CODIMENSION AND UNIFORM ALGEBRAS
}

\author{
TAKAHIKO NAKAZI* \\ Department of Mathematics, Hokkaido University, Sapporo 060-0810, Japan \\ e-mail:nakazi@math.sci.hokudai.ac.jp \\ and TOMOKO OSAWA \\ Mathematical and Scientific Subjects, Asahikawa National College of Technology, \\ Asahikwa 071-8142, Japan \\ e-mail: ohsawa@asahikawa-nct.ac.jp
}

(Received 10 October, 2002; accepted 23 June, 2003)

\begin{abstract}
Let $A$ be a uniform algebra on a compact Hausdorff space $X$ and $m$ a probability measure on $X$. Let $H^{p}(m)$ be the norm closure of $A$ in $L^{p}(m)$ with $1 \leq p<\infty$ and $H^{\infty}(m)$ the weak $*$ closure of $A$ in $L^{\infty}(m)$. In this paper, we describe a closed ideal of $A$ and exhibit a closed invariant subspace of $H^{p}(m)$ for $A$ that is of finite codimension.
\end{abstract}

2000 Mathematics Subject Classification. 46 J 15, 46 J 20.

1. Introduction. Let $A$ be a uniform algebra on a compact Hausdorff space $X$. $M(A)$ denotes the maximal ideal space of $A$. Let $m$ be a probability measure on $X . H^{p}(m)$ denotes the norm closure of $A$ in $L^{p}(m)$ with $1 \leq p<\infty$ and $H^{\infty}(m)$ denotes the weak $*$ closure of $A$ in $L^{\infty}(m) . H^{p}(m)$ is called an abstract Hardy space. When $A$ is a disc algebra, if $m$ is the normalized Lebesgue measure on the unit circle, $H^{p}(m)$ is the usual Hardy space and if $m$ is the normalized area measure on the unit disc, $H^{p}(m)$ is the usual Bergman space.

Let $I$ be a closed ideal of $A$. In this paper, we are interested in $I$ with $\operatorname{dim} A / I<\infty$. Then $A / I$ is called a Q-algebra. Two dimensional Q-algebras can be described easily; that is, $I=\left\{f \in A ; \phi_{1}(f)=\phi_{2}(f)=0\right\}$, where $\phi_{j} \in M(A) \quad(j=1,2)$, or $I=\{f \in$ $\left.A ; \phi(f)=D_{\phi}(f)=0\right\}$, where $\phi \in M(A)$ and $D_{\phi}$ is a bounded point derivation at $\phi$. One of the authors [3] showed that a two dimensional operator algebra on a Hilbert space is a Q-algebra. It seems to be worthwile to describe a finite dimensional Qalgebra. In Section 2, we describe an ideal $I$ with $\operatorname{dim} A / I<\infty$ using a theorem of T. W. Gamelin [2]. As a result, a finite dimensional $Q$-algebra is described.

When $M$ is a closed subspace of $H^{p}(m)$ and $A M \subset M, M$ is called an invariant subspace for $A$. In this paper, we are interested in $M$ with $\operatorname{dim} H^{p}(m) / M<\infty$. When $A$ is the polydisc algebra on $T^{n}$ and $m$ is the normalized Lebesgue measure on $T^{n}$, a finite codimensional invariant subspace $M$ in $H^{p}(m)$ was described by P. Ahern and D. N. Clark [1] using the ideals in the polynomial ring $C\left[z_{1}, \ldots, z_{n}\right]$ of finite codimension whose zero sets are contained in the polydisk $D^{n}$. In Section 3 , for an

* This research was partially supported by Grant-in-Aid for Scientific Research, Ministry of Education. 
arbitrary uniform algebra $A$ we describe a finite codimensional invariant subspace $M$ in $H^{p}(m)$ using the result in Section 2.

2. Finite codimensional ideal. Let $\phi \in M(A)$. A closed subalgebra $H$ of $A$ is a $(\phi, k)$-subalgebra if there is a sequence of closed subalgebras $A=A_{0} \supset A_{1} \supset \cdots \supset$ $A_{k}=H$ such that $A_{j}$ is the kernel of a continuous point derivation $D_{j}$ of $A_{j-1}$ at $\phi$. If $H$ is a $(\phi, k)$-subalgebra of $A$, then $H$ has finite codimension in $A$ and $M(H)=M(A)$ by [2, Lemma 9.1].

If $I$ is a closed ideal of $A$ and $A / I$ is of finite dimension, $B=C+I$ is a closed subalgebra of $A$, and $A / B$ is of finite dimension. By a theorem of T. W. Gamelin [2, Theorem 9.8], we can describe $B$ and so also $I$. Since $B$ is a special closed subalgebra of $A$ we can describe $I$ more explicitly.

THEOREM 1. If I is a closed ideal of $A$ and $A / I$ is of finite dimension, then there exists a closed subalgebra $E=E(I)$ of $A$ such that $E=\left\{f \in A: \phi_{1}(f)=\cdots=\phi_{n}(f)\right\}, 1 \leq$ $n<\infty,\left\{\phi_{j}\right\} \subset M(A)$ and

$$
I=H_{\phi}^{E} \cap \operatorname{ker} \phi,
$$

where $\phi=\phi_{j} \mid E, 1 \leq j \leq n$ and $H_{\phi}^{E}$ is a $(\phi, k)$-subalgebra with respect to $E$ for some $k$.

Proof. Put $H=I+C$; then $A / H$ is of finite dimension. By a theorem of T. W. Gamelin [2, Theorem 9.8], $H$ can be obtained from $A$ in two steps.

(i) There exist pairs of points $\psi_{j}, \psi_{j}^{\prime}, 1 \leq j \leq \ell$, in $M(A)$ such that if $E$ consists of the $f \in A$ such that $\psi_{j}(f)=\psi_{j}^{\prime}(f), 1 \leq j \leq \ell$, then $H \subset E \subset A$.

(ii) There exist distinct points $\theta_{j} \in M(E)$ and $\theta_{j}$-subalgebras $H_{j}$ of $E, 1 \leq j \leq k$, such that $H=H_{1} \cap \cdots \cap H_{k}$.

Put $\tilde{\psi}_{j}=\psi_{j}\left|E=\psi_{j}^{\prime}\right| E$ for $1 \leq j \leq \ell$; then $\tilde{\psi}_{j}$ belongs to $M(E)$. Since $I$ is an ideal of $A, I \subset \bigcap_{j=1}^{\ell} \operatorname{ker} \tilde{\psi}_{j}$. To see this, let $f \in A$ such that $\psi_{j}(f) \neq \psi_{j}^{\prime}(f)$. If $g \in I$, then $f g \in I$ but $\psi_{j}(f g) \neq \psi_{j}^{\prime}(f g)$ when $\tilde{\psi}_{j}(g) \neq 0$. This contradicts the fact that $f g \in E$. Thus $\tilde{\psi}_{j}(g)=0$. Hence $I \subset \bigcap_{j=1}^{\ell} \operatorname{ker} \tilde{\psi}_{j}$ and so $H \subseteq \bigcap_{j=1}^{\ell} \operatorname{ker} \tilde{\psi}_{j}+\ell$. By the definition of $E, \tilde{\psi}_{1}=\cdots=\tilde{\psi}_{\ell}$. Therefore $E$ has the form $E=\left\{f \in A ; \phi_{1}(f)=\cdots=\phi_{n}(f)\right\}, 1 \leq$ $n<\infty$, and $\left\{\phi_{j}\right\} \subset M(A)$.

For each $j$ with $1 \leq j \leq k, H_{j}$ is a $\theta_{j}$-subalgebra of $E$ for $\theta_{j} \in M(E)$. Hence there is a sequence of closed subalgebras $E=E_{j 0} \supset E_{j 1} \supset \cdots \supset E_{j \ell_{j}}=H_{j}$ such that $E_{j t}$ is the kernel of a continuous point derivation $D_{j t}$ of $E_{j t-1}$ at $\theta_{j}$. We shall write $E_{j \ell_{j}}=\operatorname{ker} D_{\theta_{j}}$, where $D_{\theta_{j}}$ is a derivation on $E_{j\left(\ell_{j}-1\right)}$. Then $H=\bigcap_{j=1}^{k} \operatorname{ker} D_{\theta_{j}}$ and so $I=\left\{\bigcap_{j=1}^{k} \operatorname{ker} D_{\theta_{j}}\right\} \cap$ $\operatorname{ker} \theta$, for some $\theta \in M(H)$. Suppose that $g$ is an arbitrary function in $I$. For any $j(1 \leq j \leq k)$, there exists a function $f \in E_{j\left(\ell_{j}-1\right)}$ such that $f \notin E_{j \ell_{j}}=\operatorname{ker} D_{\theta_{j}}$. Since $f g \in I$ and $D_{\theta_{j}}(g)=0, D_{\theta_{j}}(f g)=\theta_{j}(g) D_{\theta_{j}}(f)=0$ because $D_{\theta_{j}}$ is a derivation on $E_{j\left(\ell_{j}-1\right)}$. This implies that $\theta_{j}(g)=0$. Hence $I \subset \bigcap_{j=1}^{k} \operatorname{ker} \theta_{j}$. Therefore by the definition of $E, \theta_{1}=$ $\cdots=\theta_{k} \in M(E)$, and so $H_{1}=\cdots=H_{k}$. Thus $\theta_{1} \mid H=\theta$ and $I=\left(\operatorname{ker} D_{\theta_{1}}\right) \cap \operatorname{ker} \theta_{1}$. Since $I \subset \bigcap_{j=1}^{n} \operatorname{ker} \phi_{j}, I \subset\left(\operatorname{ker} \phi_{1}\right) \cap\left(\operatorname{ker} D_{\theta_{1}}\right) \cap \operatorname{ker} \theta_{1}$ and so $\phi_{1} \mid E=\theta_{1}$.

COROllary 1. If $I$ is a closed ideal of $A$ and $A / I$ is of finite dimension 2, then $I=\left\{f \in A ; \phi_{1}(f)=\phi_{2}(f)=0\right\}$, where $\phi_{j} \in M(A)(j=1,2)$ and $\phi_{1} \neq \phi_{2}$, or $I=$ $\left\{f \in A ; \phi(f)=D_{\phi}(f)=0\right\}$, where $\phi \in M(A)$, and $D_{\phi}$ is a bounded point derivation at $\phi$. 
Proof. When $\operatorname{dim} A / I=2$, by Theorem 1, $E=A$ or $E=\left\{f \in A ; \phi_{1}(f)=\phi_{2}(f)\right\}$. If $E=A$, then $H_{\phi}^{E}=\left\{f \in A ; D_{\phi}(f)=0\right\}$ and if $E=\left\{f \in A ; \phi_{1}(f)=\phi_{2}(f)\right\}$, then $H_{\phi}^{E}=E$, since $\operatorname{dim} A / H_{\phi}^{E}=1$ because $H_{\phi}^{E}=I+\ell$. This implies the corollary.

Corollary 2. If $B$ is a finite dimensional $Q$-algebra and $B_{0}=\operatorname{rad} B$ is its radical, then there exist subalgebras $B_{1}, B_{2}, \ldots, B_{k+1}$ in $B_{0}$ such that $B_{k+1}=\{0\}, \operatorname{dim} B_{j} / B_{j+1}=$ 1 and $B_{j+1}$ is an ideal of $B_{j}$ for $j=0,1, \ldots, k$. Hence $\operatorname{rad} B$ has a basis $\left\{f_{0}, f_{1}, \ldots, f_{k}\right\}$ such that $\left(f_{j}\right)^{2((k+1)-j)}=0$ for $j=0,1, \ldots, k$.

Proof. Since $B$ is a $Q$-algebra, $B=A / I$ for some uniform algebra $A$ and some closed ideal $I$ of $A$. Also, since $B$ is of finite dimension, we can apply Theorem 1 to $A$ and $I$. In the notation of Theorem $1, \operatorname{rad} B=\{f \in E ; \phi(f)=0\} / I$. Since $H_{\phi}^{E}$ is a $\phi$-subalgebra with respect to $E$, there exists a sequence of closed subalgebras $E=$ $E_{0} \supset E_{t} \supset \cdots ? \supset_{t} E_{k+1}=H_{\phi}^{E}$ such that $E_{j}$ is the kernel of a continuous point derivation $D_{j}$ of $E_{j-1}$ at $\phi$. Hence $E_{j+1} \cap \operatorname{ker} \phi$ is an ideal of $E_{j} \cap \operatorname{ker} \phi$ and $\operatorname{dim}\left\{E_{j} \cap \operatorname{ker} \phi / E_{j+1} \cap\right.$ $\operatorname{ker} \phi\}=1$. Put $B_{j}=\left(E_{j} \cap \operatorname{ker} \phi\right) / I$. Then $\operatorname{dim} B_{j} / B_{j+1}=1$ and $B_{j+1}$ is an ideal of $B_{j}$, for $j=0,1, \ldots, k$, and $B_{k+1}=\{0\}$. For each $j$, there exists $f_{j}$ such that $B_{j}=\left\langle f_{j}\right\rangle+B_{j+1}$ and then $\left\{f_{0}, f_{1}, \ldots, f_{k}\right\}$ is a basis of $\operatorname{rad} B=B_{0}$. Observe that $f_{j}^{2}$ belongs to $B_{j+1}$ because $E_{j+1}=\operatorname{ker} D_{j+1}$. Thus $\left(f_{j}\right)^{2(k+1-j)}=0$.

3. Finite codimensional invariant subspace. For a subset $S$ of $H^{p}(m),[S]_{p}$ denotes the closure of $S$ in $H^{p}(m)$.

THEOREM 2. If $M$ is an invariant subspace of $H^{p}$ with $\operatorname{dim} H^{p} / M=n<\infty$, then there exists a closed ideal of $A$ such that $\operatorname{dim} A / I=n,[I]_{p}=M$ and $I=M \cap A$. If $H_{\phi}^{E}$ is $a(\phi, k)$-subalgebra with respect to $E=E(I)$, then $\left[E_{j}\right]_{p} \supset\left[E_{j+1}\right]_{p}$ for any $j(0 \leq j \leq k-1)$ and $\operatorname{dim} H^{p} /[E]_{p}=\operatorname{dim} A / E$. Conversely, if $\operatorname{dim} A / I \stackrel{ \pm}{=} n<\infty$, then $\operatorname{dim} H^{p} /[I]_{p} \leq n$. If $\left[E_{j}\right]_{p} \supset_{t}\left[E_{j+1}\right]_{p}$, for any $j$ with $0 \leq j \leq k-1$ and $\operatorname{dim} H^{p} /[E]_{p}=\operatorname{dim} A / E$, then $\operatorname{dim} H^{p} /[I]_{p}=n$ and $[I]_{p} \cap A=I$.

Proof. Suppose that $M$ is an invariant subspace of $H^{p}(m)$ and $\operatorname{dim} H^{p}(m) / M=$ $n<\infty$. Then there exist $n$ linearly independent linear functionals $\psi_{1}, \ldots, \psi_{n}$ in $\left(H^{p}\right)^{*}$ such that $\psi_{j}=0$ on $M$ for $1 \leq j \leq n$. Put $\phi_{j}=\psi_{j} \mid A$ for $1 \leq j \leq n$ and $I=M \cap A$. Then $I=\bigcap_{j=1}^{n} \operatorname{ker} \phi_{j}$ and so $\operatorname{dim} A / I=n$. For $\phi_{1}, \ldots, \phi_{n}$ are independent linear functionals in $A^{*}$ because $A$ is dense in $H^{p}(m)$. If $M \supset[I]_{p}$, then there exists $\psi_{n+1} \in H^{p}(m)^{*}$ such that $\psi_{n+1}=0$ on $[I]_{p}$ and $\psi_{1}, \ldots, \psi_{n}, \psi_{n+1}$ are independent linear functionals in $\left(H^{p}\right)^{*}$. If we put $\phi_{n+1}=\psi_{n+1} \mid A$, then $\phi_{1}, \ldots, \phi_{n}, \phi_{n+1}$ are independent linear functionals in $A^{*}$ and $I \subseteq \bigcap_{j=1}^{n+1} \operatorname{ker} \phi_{j}$. This contradiction implies that $M=[I]_{p}$. Note that $\operatorname{dim} H^{p} /\left[E_{k}\right]=$ $\operatorname{dim} H^{p} /[I]_{p}-1=\operatorname{dim} A / I-1=\operatorname{dim} A / E_{k}$. If $\operatorname{dim} H^{p} /\left[E_{0}\right]_{p}<\operatorname{dim} A / E_{0}$ where $E_{0}=$ $E$ or $\left[E_{j}\right]_{p}=\left[E_{j+1}\right]_{p}$, for some $j(0 \leq j \leq k-1)$, then this contradicts the fact that $\operatorname{dim} H^{p} /\left[E_{k}\right]_{p}=\operatorname{dim} A / E_{k}$. The converse is clear.

COROllary 3. If $M$ is an invariant subspace of $H^{p}$ with $\operatorname{dim} H^{p} / M=2$, then $M=\left\{f \in H^{p} ; \Phi_{1}(f)=\Phi_{2}(f)=0\right\}$, where $\Phi_{j} \in\left(H^{p}\right)^{*}$, and $\Phi_{j}(f g)=\Phi_{j}(f) \Phi_{j}(g)$, for $f \in H^{p}$ and $g \in A$, or $M=\left\{f \in H^{p} ; \Phi(f)=D_{\phi}(f)=0\right\}$, where $\Phi, D_{\Phi} \in\left(H^{p}\right)^{*}$, $\Phi(f g)=\Phi(f) \Phi(g)$ and $D_{\Phi}(f g)=\Phi(f) D_{\Phi}(g)+\Phi(g) D_{\Phi}(f)$ for $f \in H^{p}$ and $g \in A$.

Proof. This follows from Corollary 1 and Theorem 2. 
COROLlaRY 4. If $M$ is an invariant subspace of $H^{p}$ with $\operatorname{dim} H^{p} / M=n<\infty$, then there exist $f_{1}, \ldots, f_{n}$ in $A$ such that $\left\{f_{j}+M\right\}_{j=1}^{n}$ is a basis in $H^{p} / M$.

Proof. By Theorem 2, if $I=M \cap A$, then $\operatorname{dim} A / I=n$ and $M=[I]_{p}$. Hence there exist $f_{1}, \ldots, f_{n}$ in $A$ such that $\left\{f_{j}+I\right\}_{j=1}^{n}$ is a basis in $A / I$. If $f_{j}$ belongs to $M$, then $f_{j}$ also belongs to $M \cap A=I$ and so $f_{j}$ does not belong to $M$. This proves the corollary.

\section{REFERENCES}

1. P. Ahern and D. N. Clark, Invariant subspaces and analytic continuation in several variables, J. Math. Mech. 19 (1969/1970), 963-969.

2. T. W. Gamelin, Embedding Riemann surfaces in maxinal ideal spaces, J. Funct. Anal. 2 (1968), 123-146.

3. T. Nakazi, Two-dimensional $Q$-algebras, Linear Algebra Appl. 315 (2000), 197-205. 